\title{
Free and Forced Convective Heat Transfer through a Nanofluid with Two Dimensions past Stretching Vertical Plate
}

\author{
B.Sailaja ${ }^{1}$, G. Srinivas ${ }^{2}$, B. Suresh Babu ${ }^{3}$ \\ ${ }^{1}$ Research Scholar, Dravidian University, Kuppam 517425, Telangana, India \\ ${ }^{2}$ Dept. of H\&S, Guru Nanak Institute of Technology, Hyderabad 500009, Telangana, India \\ ${ }^{3}$ Dept. of H\&S, Sreyas Institute of Engineering and Technology, Hyderabad 500068, Telangana, \\ India
}

Received: 20 April 2020; Received in revised form: 17 July 2020; Accepted: 27 July 2020;

Published online 8 August 2020

(C) Published at www.ijtf.org

\begin{abstract}
The present study focus on both free and forced convective heat transfer through a nanofluid in two dimensions past stretching vertical plate. This free and forced convective heat transfer in $\mathrm{Cu}$-water Nanofluid past permeable flat vertical semi-infinite plate was due to high conductivity and its occurrence. In this paper magnetic field and also heat source were considered. In graphs the effect on various parameters such as Reynolds number $(R e)$, solid volume fraction $(\varphi)$, magnetic field parameter $(M)$, inclination angle of the plate $(\gamma)$, heat source parameter $(Q h)$, on linear velocity $(U)$, vertical velocity $(V)$ and temperature $(\theta)$ were exhibited. The profile of every governing parameter is displayed for natural as well as forced convection by considering the $A r>>1$ and $A r<<1$ respectively. This rate of heat transfer in forced convection is more than equivalent in free convection. So these problems have several applications in engineering and petroleum industries such as electroplating, chemical processing of heavy metals and solar water heaters. Inertial force reducing the heat transfer rate in natural convection but the enhancement of $N u$ observed in forced convection. The composition of metal particles enhances the heat transfer rate in both convections, which emphasizes the nanofluid significance. Lorentz force is enhancing the heat transfer rate slightly. Heat source obviously increase the rate of heat transfer in both convections. The present paper aims to study the convective high temperature transfer of nanofluids into which viscosity proposed by Einstein and thermal conductivity proposed by Corcione were used.
\end{abstract}

Keywords: Viscosity; Thermal conductivity; Heat source; MHD; Convection; Thermal slip; Runge- kutta-6th order method; Stretching vertical plate.

\section{Introduction}

From latest record of the fluid dynamics a number of applications in several engineering problems due to a stretching sheet has gained interest in various applications which includes 
B.Sailaja et al.

International Journal of Thermofluid Science and Technology (2020), Volume 7, Issue 3, Paper No. 070302

aerodynamic extrusion of plastic sheets, the boundary layer along a liquid film in condensation process, the cooling of metallic plate in a cooling bath, glass and fiber industries and polymer extrusion in which extradite emerges from a narrow slit. The study of two-dimensional fluid flow over a linearly stretching surface was first investigated by Crane [1]. In later stages these problems were extensively studied in various directions for example non-newtonian fluids, porous space and magneto hydrodynamics etc. Some recent investigations were presented interestingly by Gupta et.al [2], the physical situations were clearly discussed by Chen and Char [3]. Another important physical phenomenon is the difference between the surface temperature and the free stream temperature is appreciably large. Hence, the conclusion for such a physical phenomenon will have a definite bearing on the plastic, fabric and polymer industries. So it is interesting to study the effects of thermal buoyancy and the Prandtl number parameter. In fact, the buoyancy force stem from the heating or cooling of a continuous stretching sheet differ the flow and the thermal fields, and thereby heat transfer characteristics of the manufacturing procedure but such effects were not considered in the study. The effects of the thermal buoyancy force on the newtonian / nonnewtonian fluid flow and heat transfer over a stretching sheet were reported by many investigators as explained in [4-11]. In addition to that both free and forced convection of heat transfer with a stretching sheet maintained at a variable temperature, this study was investigated by Vajravelu [13]. The other related analyses were performed numerically by Chen and Strobel [14] and Moutsoglou [15] for newtonian fluids under various physical situations. The influence of convective boundary conditions on hydro-magnetic mixed convection with heat and mass transfer past a vertical plate embedded in a porous medium were recently studied by Makinde and Aziz [16]. By observing all the above analysis we restricted to boundary layer flow and heat transfer in newtonian or non-newtonian fluid with a stretching sheet.

In recent years due to budding importance of nanofluids interest has been given to the study of convective heat transfer of nanofluids because the thermal conductivity of these fluids play an important role on the heat transfer coefficient between the heat transfer medium, the heat transfer surface and the convective heat transfer rate is poor in fluids such as oil, water and ethylene glycol mixture. Kakaç and Pramuanjaroenkij [17] have developed through various methods, which are proposed to improve the thermal conductivity of these fluids. In order to develop advanced heat transfer fluid with significantly higher conductivities, they are first initiated by Choi [18] this is an innovative technique which is used in a mixture of nanoparticles and the base fluid. By having unique physical and chemical properties it is referred and then nanofluid which is resulting a mixture of the base fluid and nanoparticles. A liquid suspension containing ultra-fine particles with diameter less than $50 \mathrm{~nm}$ is described in nanofluid. A remarkable improvement in the convective heat transfer coefficient experimental studies shows that even with small volumetric fraction of nanoparticles usually less than 5\% the thermal conductivity of the base fluid is enhanced through 10-50\% this phenomenon observed by Masuda et al. [19] by the characteristic feature of nanofluid in the thermal conductivity enhancement. The possible uses of nanofluids in advanced nuclear systems were suggested by Buongiomo and $\mathrm{Hu}$ [20]. Recently the study of flow and heat transfer is studied by Bachok et al. [21] in an incompressible viscous fluid near the three-dimensional stagnation point of a body and it is placed in a water based nanofluid containing different types of nanoparticles. The available literature on nanofluids with heat transfer shows that the work is not carried out for viscous 
B.Sailaja et al.

International Journal of Thermofluid Science and Technology (2020), Volume 7, Issue 3, Paper No. 070302

fluid flow and heat transfer over a continuously moving surface. The number of experimental and numerical studies on convection flow in nanofluids (reported in the literature), still there is a lack of information in the problem of heat transfer enhancement over a stretching sheet with nanofluids. The best knowledge of the authors information no studies have been reported in the literature, this problem may be encountered in a number of electronic cooling devices equipped through nanofluids. The boundary layer and the Boussinesq approximations are used to simplify the momentum and the energy equations assuming that the base fluids and their physical/rheological properties were taken constant. The focus of the present study is to analyze the effect of several pertinent parameters such as the free convection parameter which depend on the temperature difference, nanoparticle size volume fraction and the internal heat source/sink parameter on the flow and heat transfer characteristics with two different types of nanofluid particles which are namely copper-water and silver-water case so the momentum and energy equations were coupled and they are non-linear. By using similarity transformation these coupled and nonlinear equations are transformed into coupled equations by using various methods.

In view of the boundary layer flow on the stretching sheet which plays a significant role in the application areas namely aerodynamics, plastic sheet extrusion, metal whirling, plastic producer, rubber sheet producer, document production and so on thus it remains on the leading boundary of the machinery improvement. For industrialized operations these metals were most commonly used alloys which heats up till it was molted, whereas it was decant interest in a molded material otherwise it expires which will also contain a cavity of necessary shape. This discovery of convective Nanofluid dominates the heat transfer throughout the fluid with its physically powerful property of matter (solid along with its base fluid). Thus the existing literature analysis explains how to study the Nanofluid heat transfer by its value and it is sufficient to execute within the industry. Sarith Kumar Das [22] along with Xiang- Qi Wang [23] both are stressed out mainly on the need of heat transfer mechanism throughout Nanofluid, in addition to its interaction of metal particle by means of the solvent. Further many other instigators endeavor to learn the thermal conductivity and also viscosity through their physical parameter. Tiwari Arun Kumar [24] studies the thermal conductivity through viscosity of Nanofluid by means of a variety of models and also he completed existing literature and it was still ambiguous towards match trial and hypothetical consequences. In recent times Gbadeyan [25] reported to enhance inside Brownie motion which reduces the heat transfer speed. On the other hand, Hamad [26] completed the Nanoparticle enclosure change flow patron significantly in the base fluid. Again to match trial and hypothetical consequences Hassan [27] along with Srikanth [28] attempted the diffusion equation which includes the chemical reaction coefficients. An effort was made at this point to scrutinize the convective heat as well as mass transfer of Nanofluid through earlier period stretch sheet. Now in this chapter we discussed about free and forced convection heat transfer through a Nanofluid in two dimensions past stretching vertical plate. At the outset, the heating or cooling systems efficiency has increased by introducing nano fluid. But in practice cooling or heating systems are designed as free or forced convection systems. When porous medium is present in the flow region, the study of forced or free convection study is more relevant in real time systems.

The focus of the present study is to analyze the effect of free and forced convection of copper-water nanofluid past a stretching sheet. Porous medium, Magnetic field and 
B.Sailaja et al.

International Journal of Thermofluid Science and Technology (2020), Volume 7, Issue 3, Paper No. 070302

temperature dependent heat source are considered. The governing equations are coupled and are solved numerically.

\section{Formulation of the Problem}

The main assumption governing the flow and heat transfer is as follows:

$>$ Consider Copper water nanofluid flow past a moving semi infinite flat plate.

$>$ The nanofluid flow was assumed in the x.direction.

$>$ Initially entire system is at rest.

$>$ This plate was assumed to be non-electrically conducted, exponentially stretched along $\mathrm{x}$ and y directions and move in the normal direction with a velocity $\left[U_{0}\right]$.

$>$ Suppose the regular fluid and suspended $\mathrm{Cu}$ particle both are at thermal equilibrium and no slip occur between them.

Transverse magnetic field was applied uniformly.

$>$ It was assumed that magnetic field induced was lesser than magnetic field applied externally.

$>$ By taking surface temperature at constant value $T_{w}$ and the ambient temperature $T_{\infty}$ where $T_{w}>T_{\infty}$

$>$ The fluid is assumed to follow the Boussinesq approximation

The Cartesian co-ordinate system and also the geometry of the plate are shown in the following diagram.

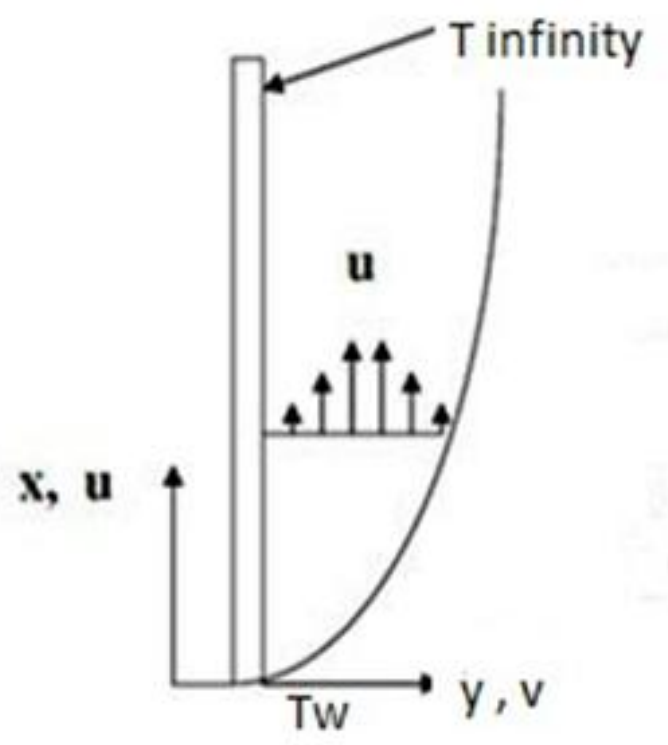

Fig 1 Schematic diagram 
B.Sailaja et al.

International Journal of Thermofluid Science and Technology (2020), Volume 7, Issue 3, Paper No. 070302

\section{Governing Equations}

The boundary layer equation governing the flow and temperature (Under the application of Bossinesq approximations as well as boundary layer approximations) as per above assumption are as follows

$$
\begin{aligned}
& \frac{\partial u}{\partial x}+\frac{\partial v}{\partial y}=0 \\
& \rho_{n f}\left(\frac{\partial u}{\partial t}+u \frac{\partial u}{\partial x}+v \frac{\partial u}{\partial y}\right)=\mu_{n f}\left(\frac{\partial^{2} u}{\partial x^{2}}+\frac{\partial^{2} u}{\partial y^{2}}\right)+\left(\rho \beta_{T}\right)_{n f} g\left(T-T_{\infty}\right)-\sigma B_{0}^{2} u \\
& \rho_{n f}\left(\frac{\partial v}{\partial t}+u \frac{\partial v}{\partial x}+v \frac{\partial v}{\partial y}\right)=\mu_{n f}\left(\frac{\partial^{2} v}{\partial x^{2}}+\frac{\partial^{2} v}{\partial y^{2}}\right) \\
& \frac{\partial T}{\partial t}+u \frac{\partial T}{\partial x}+v \frac{\partial T}{\partial y}=\alpha_{n f}\left(\frac{\partial^{2} T}{\partial x^{2}}+\frac{\partial^{2} T}{\partial y^{2}}\right)-\frac{Q}{\left(\rho c_{p}\right)_{n f}}\left(T-T_{\infty}\right)
\end{aligned}
$$

The boundary condition for this problem is as follows

$$
\begin{aligned}
& u(x, y, t)=0, v(x, y, t)=0, T(x, y, t)=T_{\infty}, t<0 \\
& u(0, y, t)=U_{0}\left(1+e^{\frac{y}{L}}\right), v(0, y, t)=U_{0}\left(1+e^{\frac{y}{L}}\right), T(o, y, t)=T_{w}, y>0, t \geq 0 \\
& u(x, 0, t)=U_{0}\left(1+e^{\frac{x}{L}}\right), v(x, 0, t)=U_{0}\left(1+e^{\frac{x}{L}}\right), T(x, 0, t)=T_{w}, x>0, t \geq 0 \\
& u(\infty, y, t) \rightarrow 0, v(\infty, y, t) \rightarrow 0, T(\infty, y, t) \rightarrow T_{\infty}, t \geq 0 \\
& u(x, \infty, t) \rightarrow 0, v(x, \infty, t) \rightarrow 0, T(x, \infty, t) \rightarrow T_{\infty}, t \geq 0
\end{aligned}
$$

Thermo-Physical properties were related as follows:

$$
\begin{aligned}
& \rho_{n f}=(1-\phi) \rho_{f}+\phi \rho_{s}, \alpha_{n f}=\frac{k_{n f}}{\left(\rho c_{p}\right)_{n f}} \\
& \left(\rho c_{p}\right)_{n f}=(1-\phi)\left(\rho c_{p}\right)_{f}+\phi\left(\rho c_{p}\right)_{s} \\
& (\rho \beta)_{n f}=(1-\phi)(\rho \beta)_{f}+\phi(\rho \beta)_{s} \\
& \mu_{r}=\frac{\mu_{n f}}{\mu_{f}}=1+2.5 \phi \\
& \frac{k_{n f}}{k_{f}}=1+4.4 \operatorname{Re}^{0.4} \operatorname{Pr}^{0.66}\left(\frac{T}{T_{f r}}\right)^{10}\left(\frac{k_{p}}{k_{f}}\right)^{0.03} \phi^{0.66}
\end{aligned}
$$

Here we introduced the following dimensionless variables as follows

$$
X=\frac{x}{L}, Y=\frac{y}{L}, t^{\prime}=t \frac{U_{0}}{L}, U=\frac{u}{U_{0}}, V=\frac{v}{U_{0}}, \theta=\frac{\mathrm{T}-\mathrm{T}_{\infty}}{\mathrm{T}_{w}-\mathrm{T}_{\infty}}
$$

Equations (2), (3) and (4) can be written in the following dimensionless form by using equations (6), (7), (8) 


$$
\begin{aligned}
& \frac{\partial U}{\partial t}+U \frac{\partial U}{\partial X}+V \frac{\partial U}{\partial Y}=\frac{1+2.5 \phi}{\left(1-\phi+\phi\left(\frac{\rho_{s}}{\rho_{f}}\right)\right)} \frac{1}{\operatorname{Re}}\left(\frac{\partial^{2} U}{\partial X^{2}}+\frac{\partial^{2} U}{\partial Y^{2}}\right)+\left(1-\phi+\phi\left(\frac{\beta_{s}}{\beta_{f}}\right)\right) \frac{G r}{R^{2}} \theta-M^{2} U \\
& \frac{\partial V}{\partial t}+U \frac{\partial V}{\partial X}+V \frac{\partial V}{\partial Y}=\frac{1+2.5 \phi}{\left(1-\phi+\phi\left(\frac{\rho_{s}}{\rho_{f}}\right)\right)} \frac{1}{\operatorname{Re}}\left(\frac{\partial^{2} V}{\partial X^{2}}+\frac{\partial^{2} V}{\partial Y^{2}}\right) \\
& \frac{\partial \theta}{\partial t}+U \frac{\partial \theta}{\partial X}+V \frac{\partial \theta}{\partial Y}=\frac{1+4.4 \operatorname{Re}^{0.4} \operatorname{Pr}^{0.66}\left(\frac{T}{T_{f r}}\right)^{10}\left(\frac{k_{s}}{k_{f}}\right)^{0.03} \phi^{0.66}}{\left(1-\phi+\phi\left(\frac{\left(\rho c_{p}\right)_{s}}{\left(\rho c_{p}\right)_{f}}\right)\right)} \frac{1}{\operatorname{Pr}} \frac{1}{\operatorname{Re}}\left(\frac{\partial^{2} \theta}{\partial X^{2}}+\frac{\partial^{2} \theta}{\partial Y^{2}}\right)- \\
& \frac{Q_{h}}{\left(1-\phi+\phi\left(\frac{\left(\rho c_{p}\right)_{s}}{\left(\rho c_{p}\right)_{f}}\right)\right.} \frac{1}{\operatorname{Pr}} \frac{1}{\operatorname{Re}} \theta
\end{aligned}
$$

Where the corresponding boundary condition of equation (5) are written in the dimensionless form as follows

$$
\begin{aligned}
& U\left(X, Y, t^{\prime}\right)=0, V\left(X, Y, t^{\prime}\right)=0, T\left(X, Y, t^{\prime}\right)=0, t^{\prime}<0 \\
& U\left(0, Y, t^{\prime}\right)=1+e^{Y}, V\left(0, Y, t^{\prime}\right)=1+e^{Y}, \theta\left(0, Y, t^{\prime}\right)=1, Y>0, t^{\prime} \geq 0 \\
& U\left(X, 0, t^{\prime}\right)=1+e^{X}, V\left(X, 0, t^{\prime}\right)=1+e^{X}, \theta\left(X, 0, t^{\prime}\right)=1, X>0, t^{\prime} \geq 0 \\
& U\left(\infty, Y, t^{\prime}\right) \rightarrow 0, V\left(\infty, Y, t^{\prime}\right) \rightarrow 0, \theta\left(\infty, Y, t^{\prime}\right) \rightarrow 0, t^{\prime} \geq 0 \\
& U\left(X, \infty, t^{\prime}\right) \rightarrow 0, V\left(X, \infty, t^{\prime}\right) \rightarrow 0, \theta\left(X, \infty, t^{\prime}\right) \rightarrow 0, t^{\prime} \geq 0 \\
& U(x, \infty, t) \rightarrow 0, V(x, \infty, t) \rightarrow 0, \theta(x, \infty, t) \rightarrow 0, t \geq 0
\end{aligned}
$$

The parameters present in the above equations are as follows:

Prandtl Number $\operatorname{Pr}=\frac{v_{f}}{\alpha_{f}}$, Magnetic field parameter $M^{2}=\frac{\sigma B_{0}{ }^{2} L}{U_{0} \rho_{f}}$,

Reynolds Number $\operatorname{Re}=\frac{L U_{0}}{v}$, Heat source parameter $Q_{h}=\frac{Q L^{2}}{k_{n f}}$

Grashoff Number $G r=\frac{g \beta_{T f}\left(T_{w}-T_{\infty}\right) L^{3}}{v_{f}{ }^{2}}$, Archimedes Number $A r=\frac{G r}{\operatorname{Re}^{2}}$

Biot Number $B i=\frac{h L}{k}$ and $k=\frac{M L}{T^{3} \theta}$ 
B.Sailaja et al.

International Journal of Thermofluid Science and Technology (2020), Volume 7, Issue 3, Paper No. 070302

\section{Solution to the Problem}

The differential equations from (9) to (11) were coupled and they are nonlinear. These equations are solved subjected to the boundary condition shown in (12). Domain considered is an infinite rectangular plate; the study is in two dimensions.

For computational purpose the height of the plate is considered to be of 2 units and the width of the plate is to be of 1 unit. To solve this system we used "ND Solve" tool in Mathematica 10.4

Local heat transfer rate is determined by Nusselt number $(\mathrm{Nu})$ in dimensionless form and it was given by,

$$
N u=-\frac{k_{n f}}{k_{f}} \theta^{\prime}(0)
$$

\section{Results and Discussion}

The effect on various parameter such as Reynolds number $(R e)$, solid volume fraction $(\phi)$, magnetic parameter $(M)$, inclination angle of the plate $(\gamma)$, heat source parameter ( $Q h)$ on governing parameters $U, V$ and $\theta$ were exhibited in the graphs from Figures 2 to 16 . This profile of every governing parameter is displayed for natural as well as forced convection by considering the $A r>>1$ and $A r<<1$.

For computation we assumed two cases as $A r=500$ and $A r=1 / 500$ and the other parameters are treated as constants. The Prandtl Number ( $\mathrm{Pr}$ ) is kept as constant and it is 0.7 for water. The vertical velocity ( $U$ ) and the temperature ( $\theta$ ) were calculated at $y=1 / 2$ level and the horizontal velocity $(V)$ is presented at $x=1$ level.

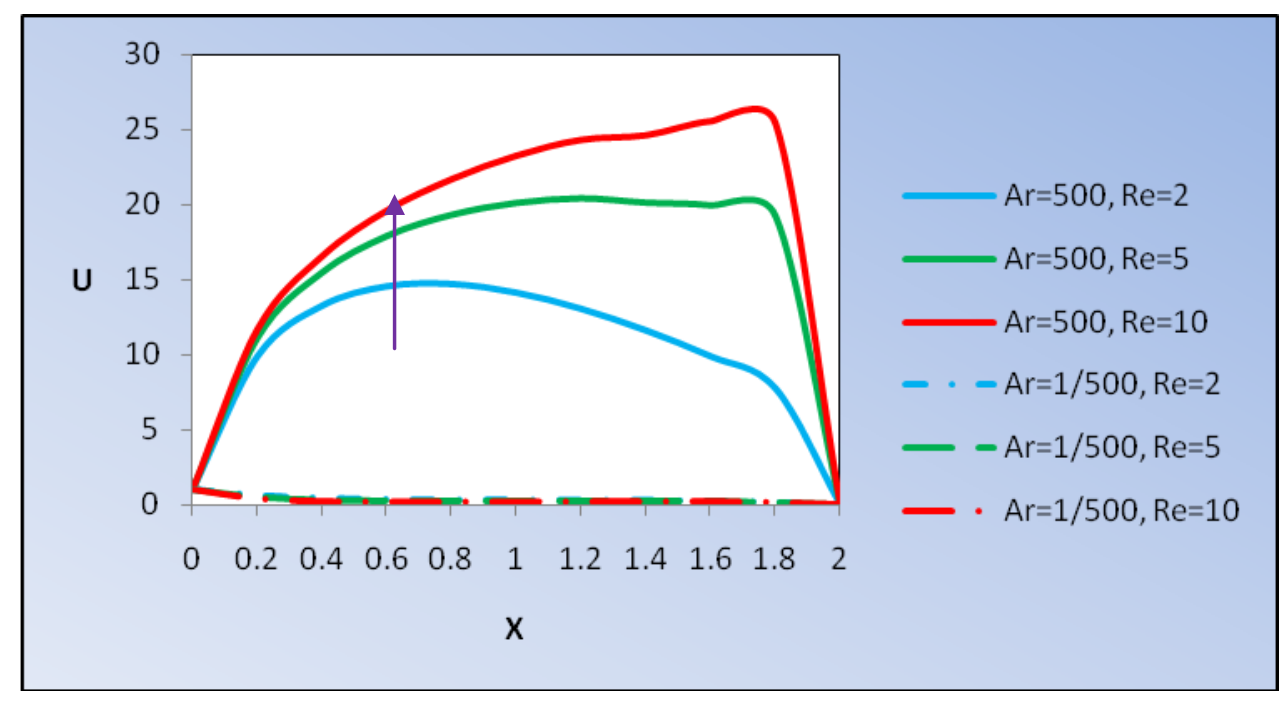

Fig.2 Profile of vertical velocity U with Re 


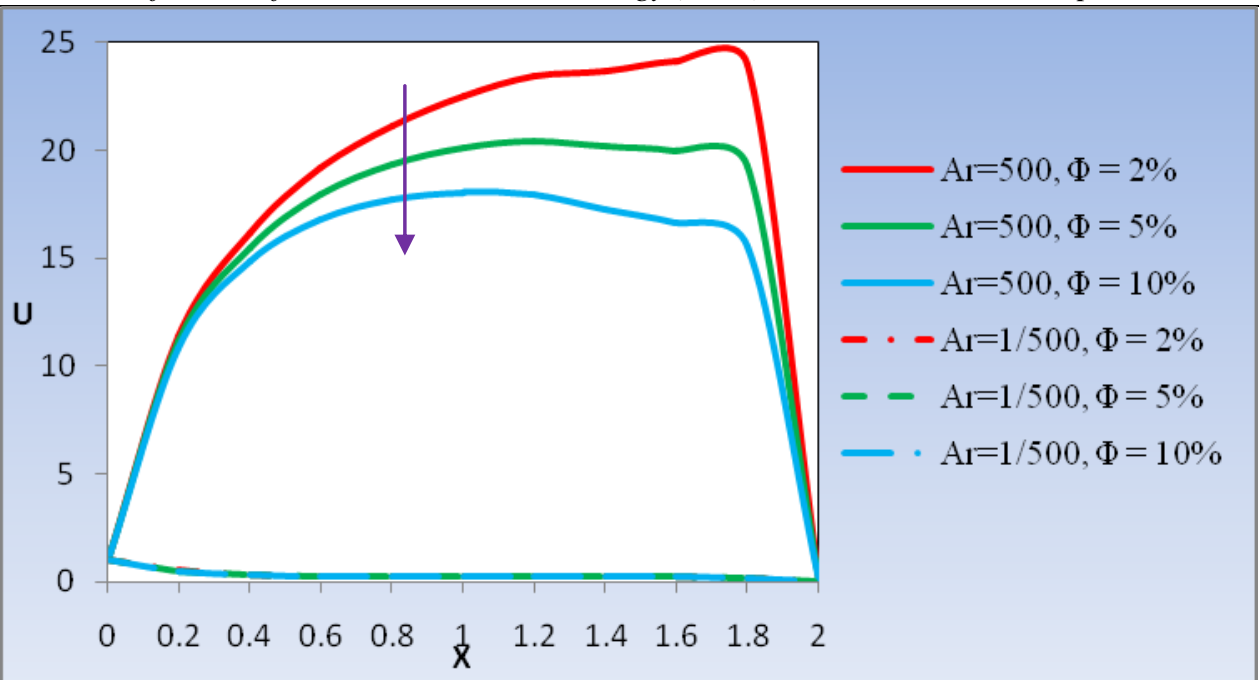

Fig.3 Profile of vertical velocity $U$ with $\varphi$

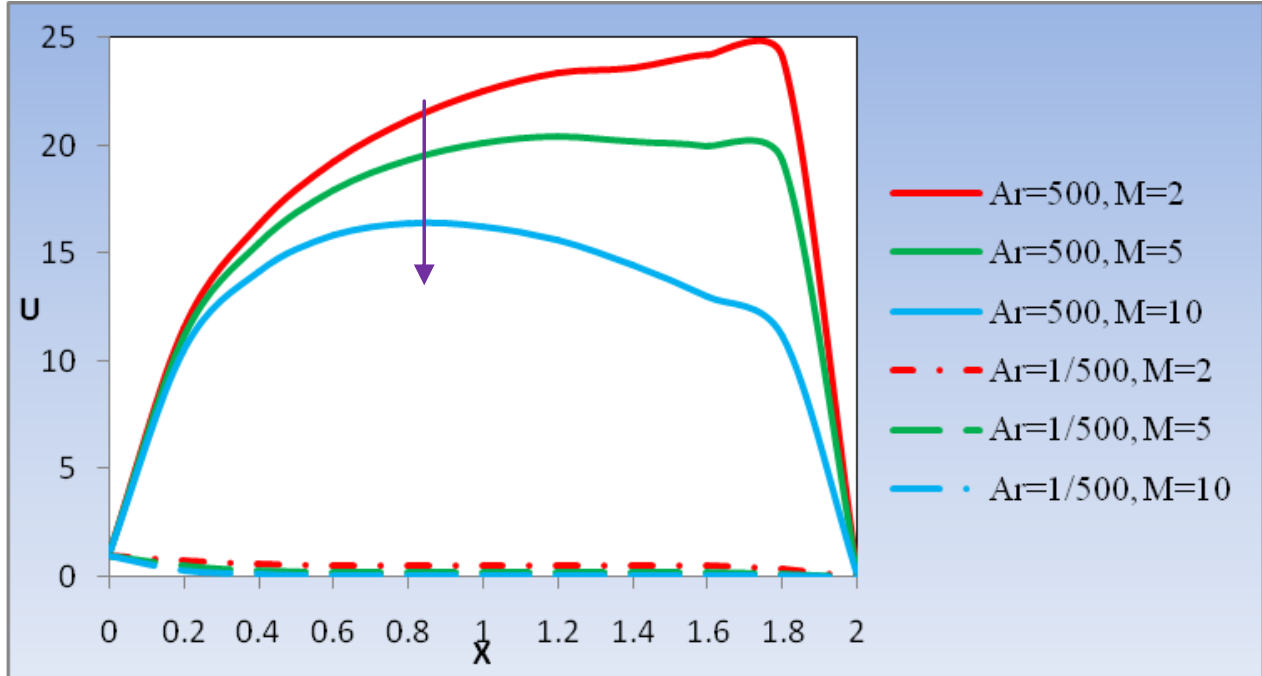

Fig.4 Profile of U with M

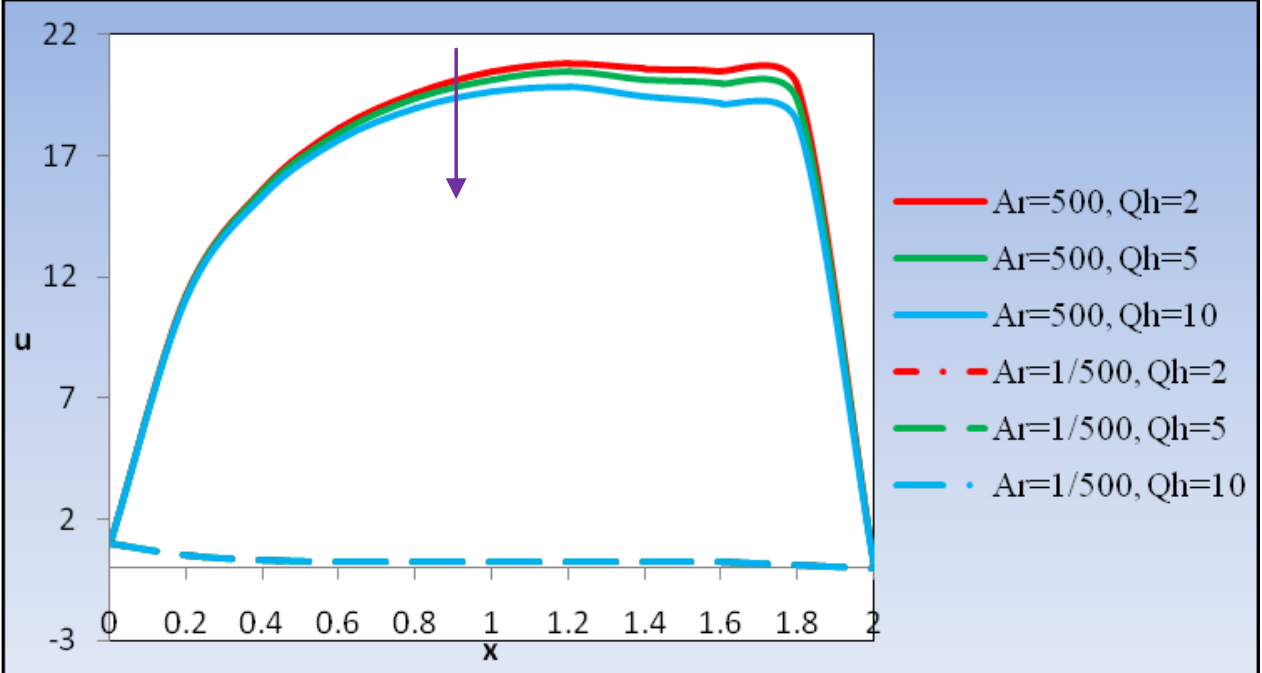

Fig.5 Profile of vertical velocity $U$ with $\mathrm{Q}_{\mathrm{h}}$ 


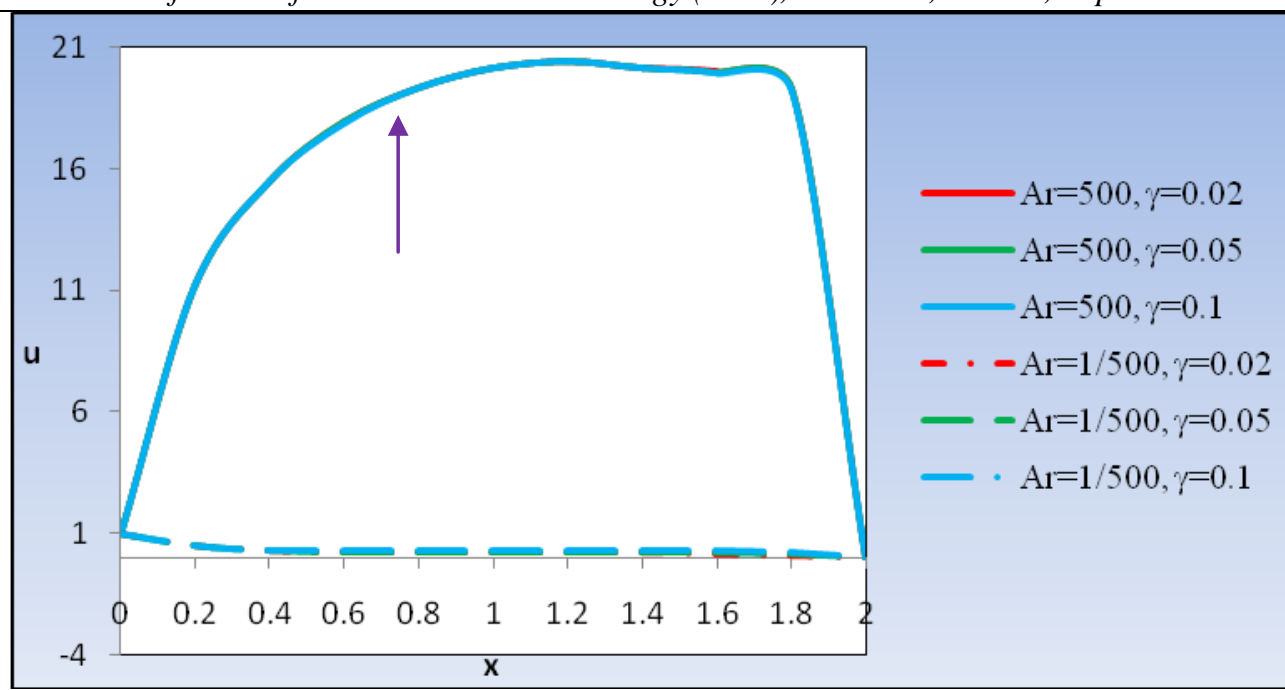

Fig.6 Profile of vertical velocity $U$ with $\gamma$

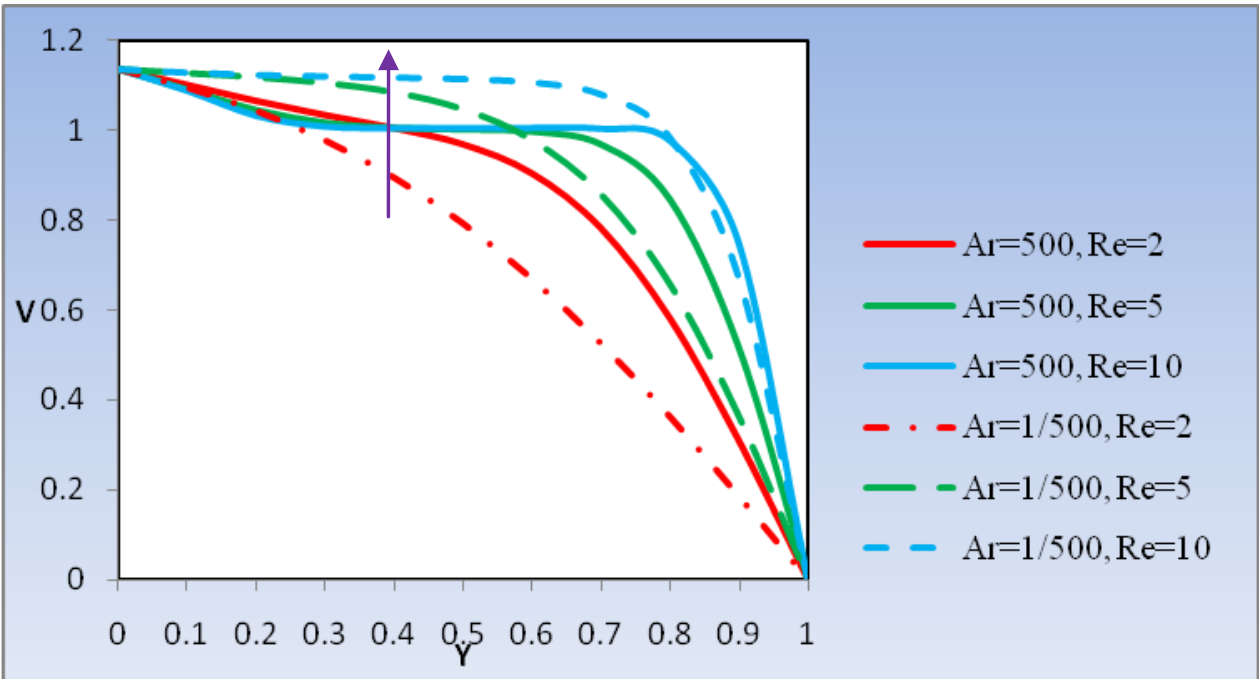

Fig.7 Profile of V with Re

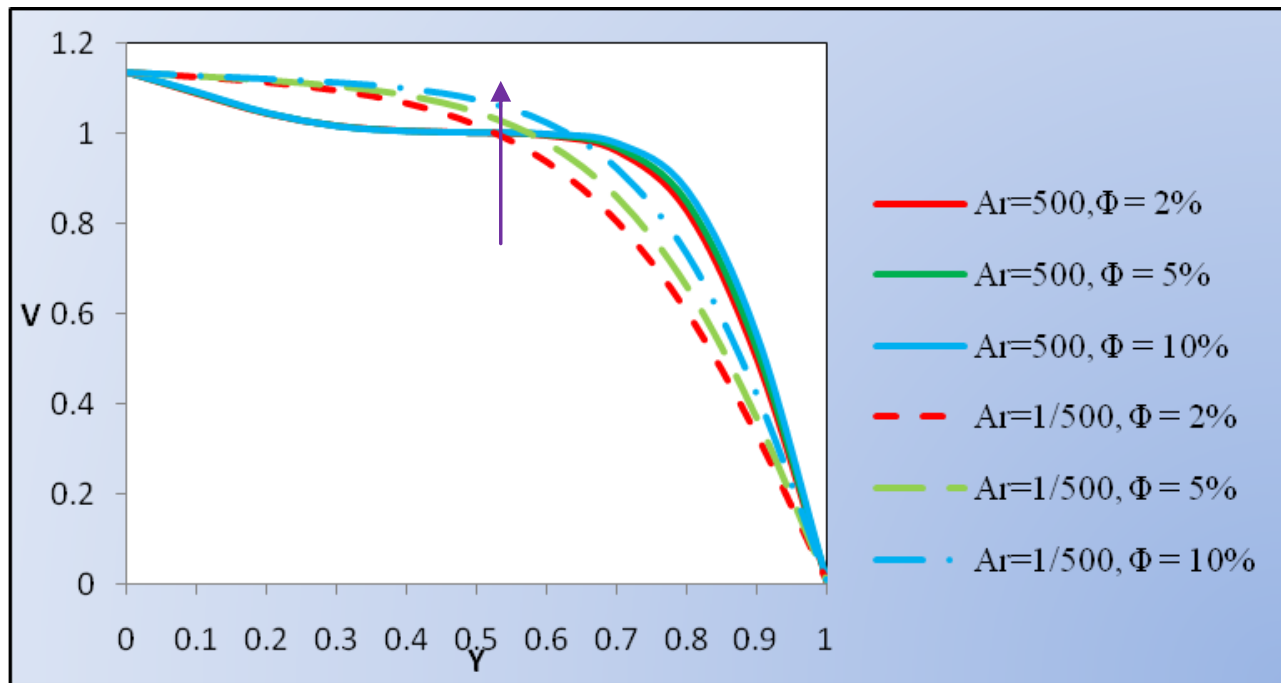

Fig.8 Profile of V with $\varphi$ 


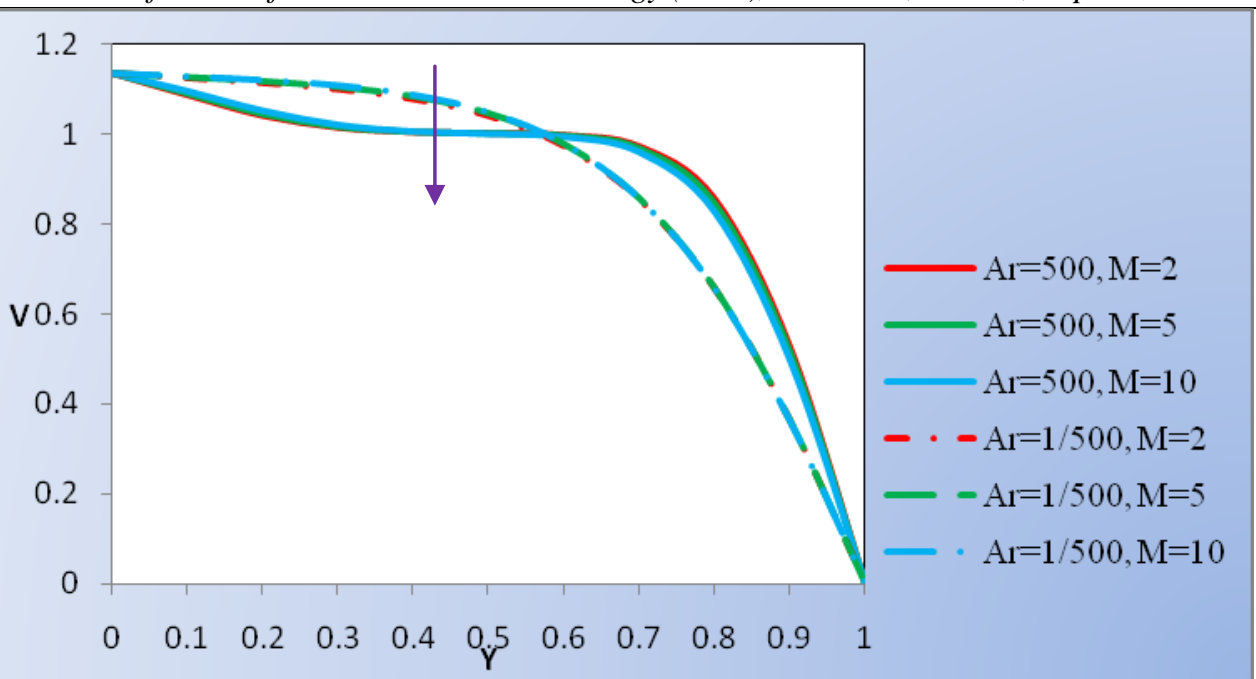

Fig.9 Profile of V with $\mathrm{M}$

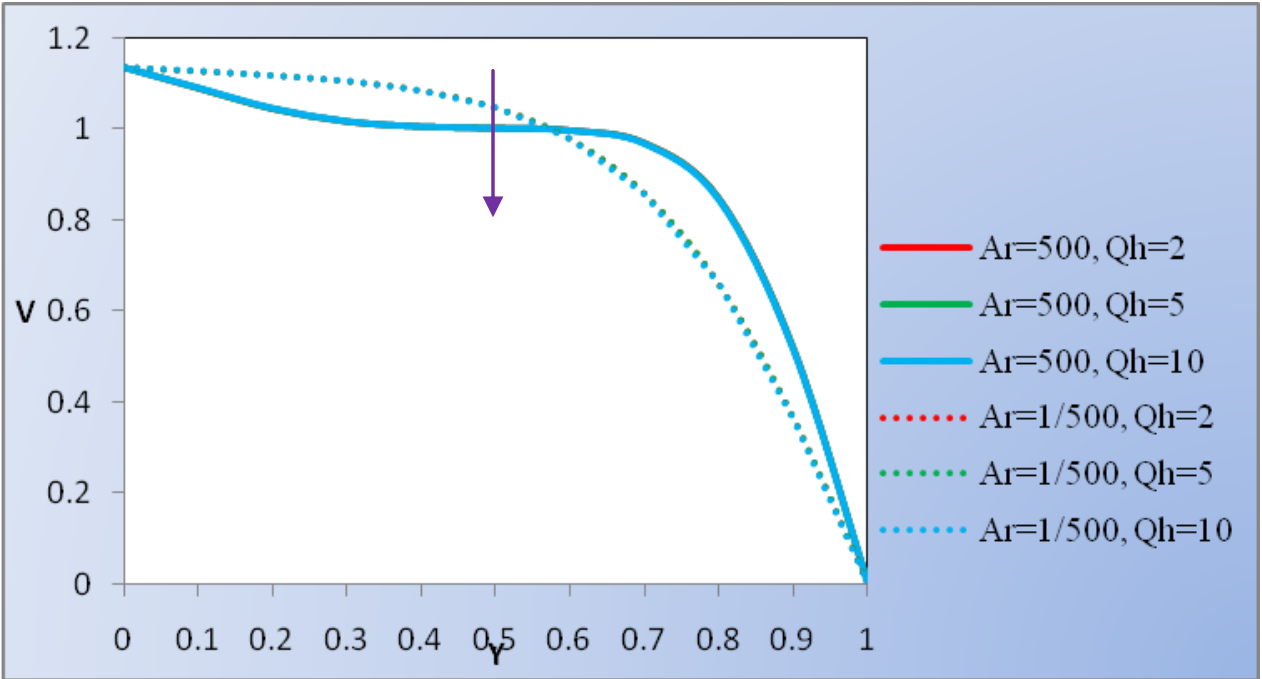

Fig. 10 Profile of $\mathrm{V}$ with $\mathrm{Q}_{\mathrm{h}}$

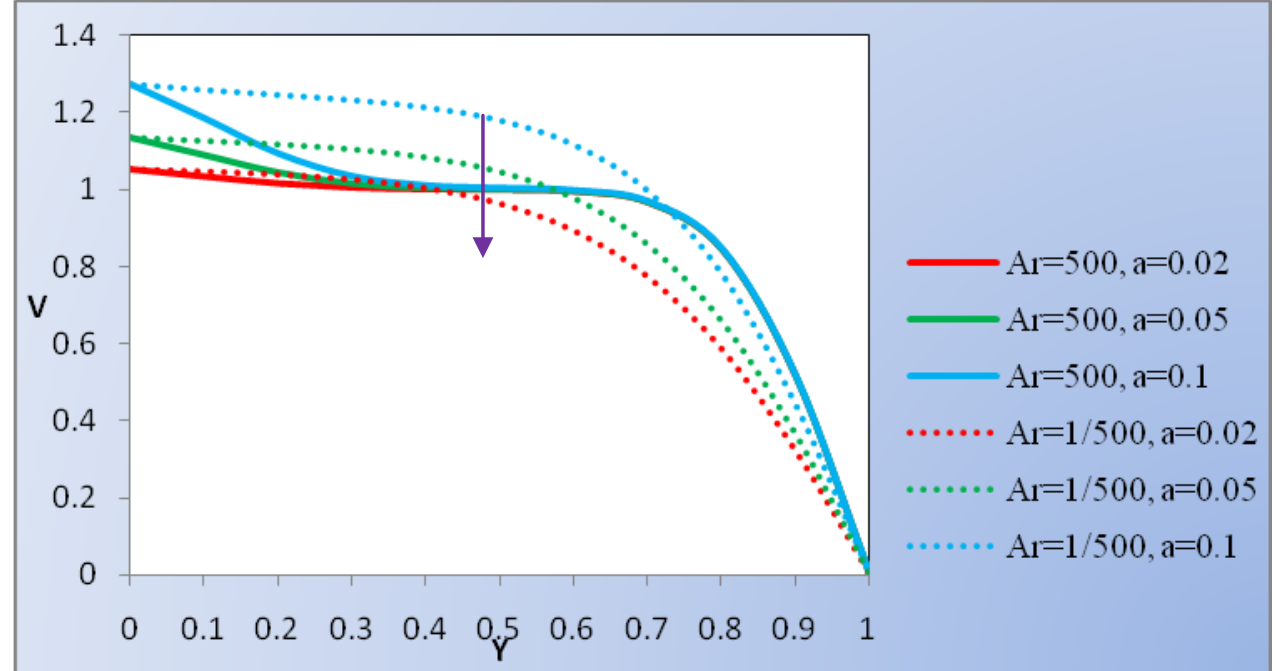

Fig.11 Profile of V with $\gamma$ 


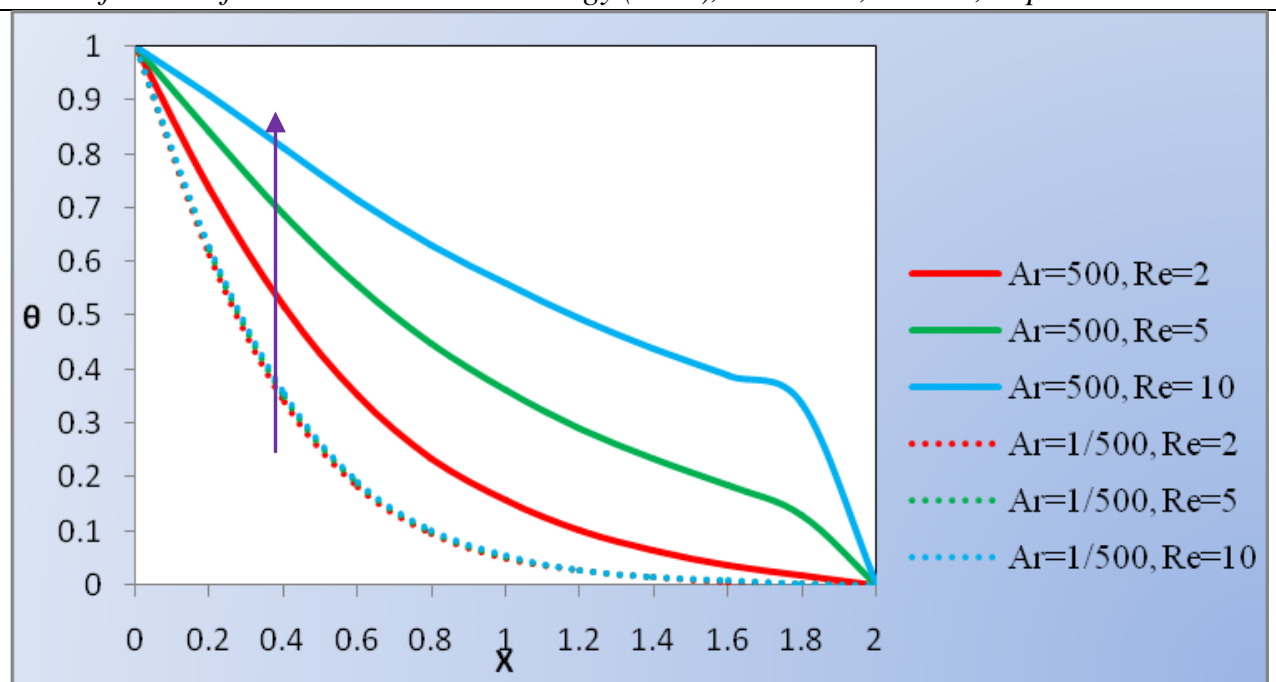

Fig.12 Profile of $\theta$ with Re

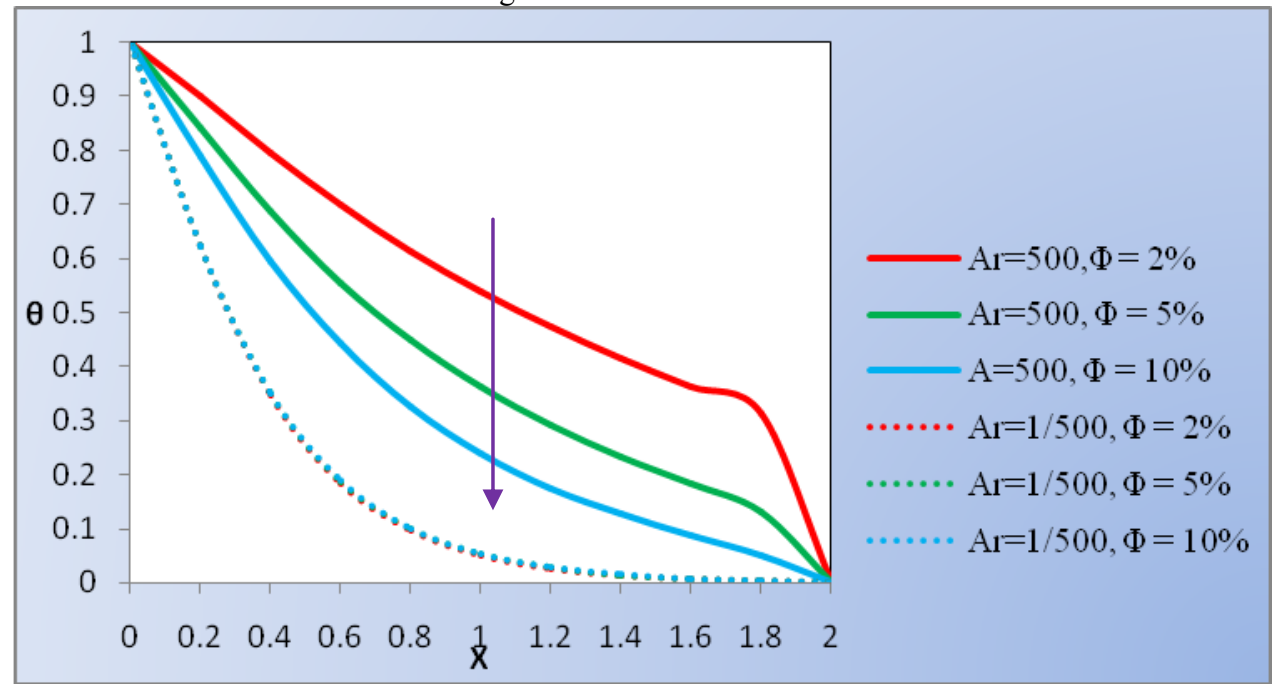

Fig.13 Profile of $\theta$ with $\varphi$

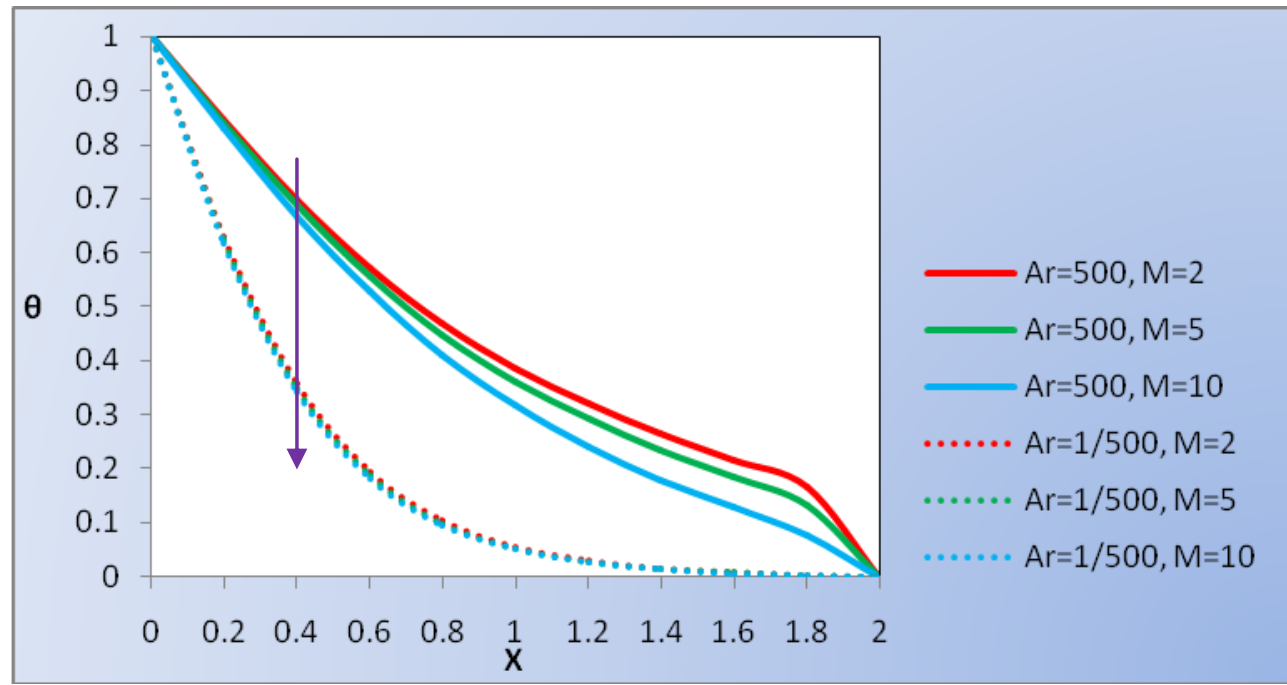

Fig.14 Profile of $\theta$ with $\mathrm{M}$ 


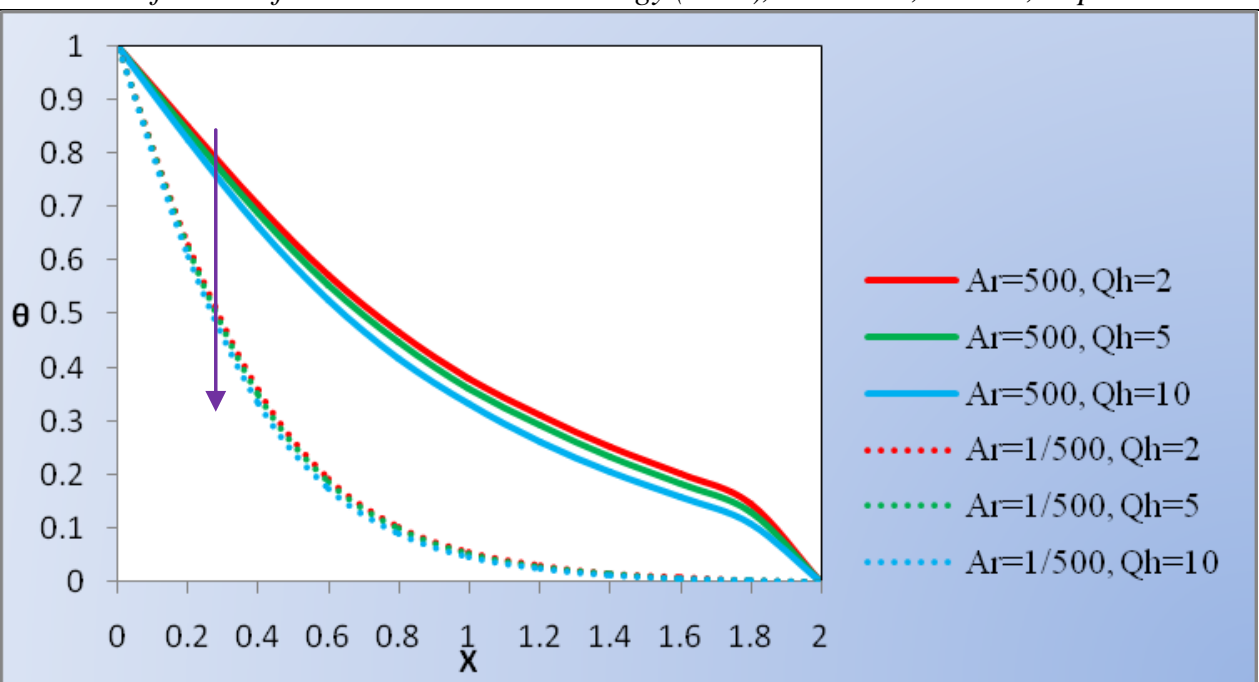

Fig. 15 Profile of $\theta$ with $Q_{h}$

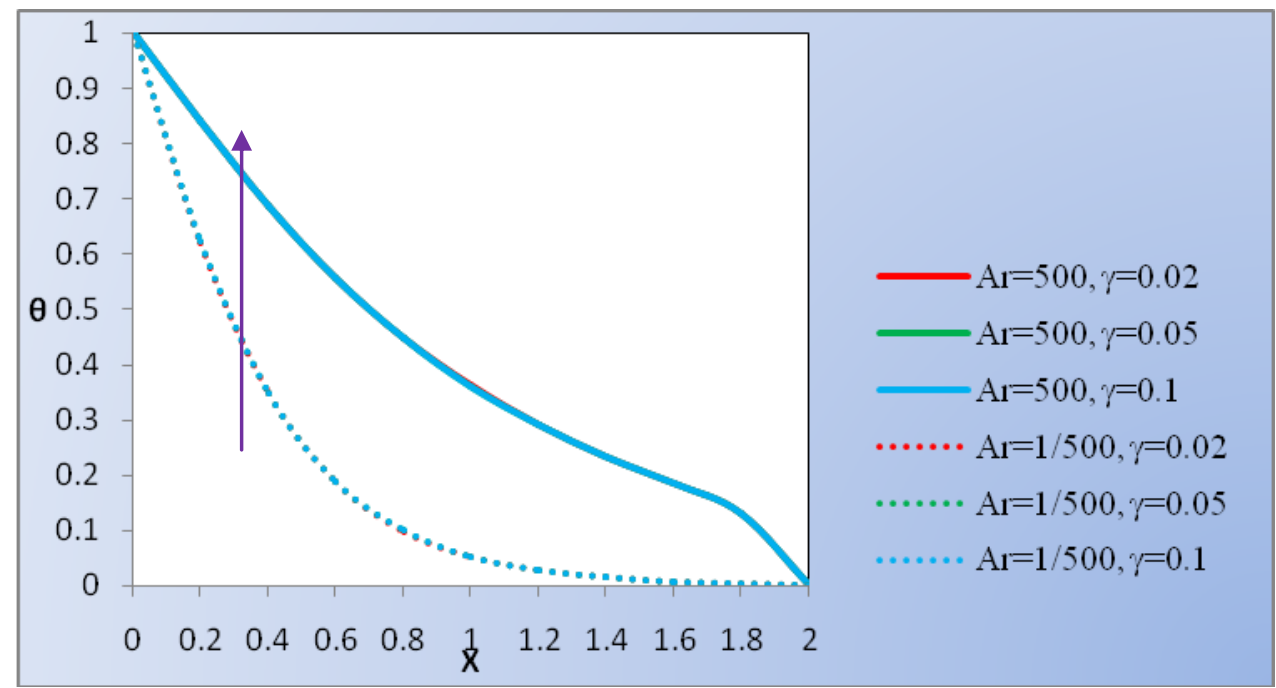

Fig.16 Profile of $\theta$ with $\gamma$

The vertical velocity $(U)$ profiles are displayed from figures 2-6. The vertical flow is found more in natural convection than in forced convection for all variations. Movement of the plate accelerates the flow more in natural convection. Thus the movement of the boundary regulates the flow in forced convection case. From Fig.2 it is evident that the flow was directly proportional to the inertial force in natural convection and the flow is inversely proportional to the inertial force in forced convection. Fig. 3 confirms that the solid volume fraction is always inversely proportional to the flow in both convections.

But the flow retards gradually. From Fig.4 Lorentz force dominates the flow in both convections. Heat source is not much significant on flow in both convections, but it showed negative impact when it was increased (Fig.5). It was happened in the presence of metal particles which act as heat absorbers. So the $\mathrm{Cu}$-water Nanofluid will be preferred in heat generating systems. From Fig. 6 it is evident that the flow is inversely proportional to the stretching rate in natural convection and the flow is directly proportional to the stretching rate in forced convection. 
B.Sailaja et al.

International Journal of Thermofluid Science and Technology (2020), Volume 7, Issue 3, Paper No. 070302

The horizontal velocity $(V)$ profiles are displayed from figures 7-11. The horizontal velocity is found more in natural convection than in forced convection for all variations. The flow was found to be more near the base of the plate and retards from the base.

From Fig.7, viscous force dominates the inertial force and hence the velocity enhances as Re increases. From Fig. 8 the presence of metal particle enhance velocity in the Brownian motion of the particle. It was further observed that variation of velocity is significant in forced convection than in natural convection. Fig.9 exhibits the reduction of velocity with Lorentz force in both the convections. But the variation of $M$ is not clearly significant in both convections. From Fig.10 the variation of $V$ with $Q h$ the heat source is slightly affecting the velocity $V$. It is found that the variation of $Q h$ reduces the velocity. From Fig.11 the variation of $V$ with $\gamma$, it is found that the increase of stretching rate enhances the velocity in both convections.

The temperature $(\theta)$ profiles are displayed from Fig.12-16. The temperature is significant for variation of different parameters in natural convection and on the other hand the forced convection was not showing significant variation of temperature. In Fig.12 the temperature increases with increase in inertial force. Fig.13 shows that solid volume fraction was inversely proportional to the temperature in natural convection and directly proportional in forced convection. It may happen due to agglomeration of metal particles. The flow retards with increase in Lorentz force retards temperature as per Fig 14. Interestingly by Fig.15 increased heat source decrease the temperature in both convections. This occurs due to Copper Nano-particles of the fluids. The slight temperature reduction with increase in Biot number in both convections is observed from Fig.16.

\begin{tabular}{cccccc}
\hline & \multicolumn{5}{c}{ Ar=500 } \\
\hline $\mathbf{R e}$ & $\varphi$ & $\mathrm{M}$ & $\mathrm{Q}_{\mathrm{h}}$ & $\gamma$ & $\mathrm{Nu}$ \\
$\mathbf{2}$ & $5 \%$ & 5 & 5 & 0.05 & 7.09271 \\
$\mathbf{5}$ & $5 \%$ & 5 & 5 & 0.05 & 5.49932 \\
$\mathbf{1 0}$ & $5 \%$ & 5 & 5 & 0.05 & 3.80862 \\
$\mathbf{5}$ & $2 \%$ & 5 & 5 & 0.05 & 2.14513 \\
$\mathbf{5}$ & $5 \%$ & 5 & 5 & 0.05 & 5.49932 \\
$\mathbf{5}$ & $10 \%$ & 5 & 5 & 0.05 & 11.177 \\
$\mathbf{5}$ & $5 \%$ & 2 & 5 & 0.05 & 5.32875 \\
$\mathbf{5}$ & $5 \%$ & 5 & 5 & 0.05 & 5.49932 \\
$\mathbf{5}$ & $5 \%$ & 10 & 5 & 0.05 & 5.79725 \\
$\mathbf{5}$ & $5 \%$ & 5 & 2 & 0.05 & 4.99571 \\
$\mathbf{5}$ & $5 \%$ & 5 & 5 & 0.05 & 5.49932 \\
$\mathbf{5}$ & $5 \%$ & 5 & 10 & 0.05 & 6.32375 \\
$\mathbf{5}$ & $5 \%$ & 5 & 5 & 0.02 & 5.49816 \\
$\mathbf{5}$ & $5 \%$ & 5 & 5 & 0.05 & 5.49932 \\
$\mathbf{5}$ & $5 \%$ & 5 & 5 & 0.1 & 5.5013 \\
\hline
\end{tabular}

Table 1- Nusselt Number $(\mathrm{Nu})$ for free convection 


\begin{tabular}{cccccc}
\hline \multicolumn{5}{c}{ Ar=1/500 } \\
\hline $\mathbf{R e}$ & $\varphi$ & $\mathrm{M}$ & $\mathrm{Q}_{\mathrm{h}}$ & $\gamma$ & $\mathrm{Nu}$ \\
$\mathbf{2}$ & $5 \%$ & 5 & 5 & 0.05 & 11.0551 \\
$\mathbf{5}$ & $5 \%$ & 5 & 5 & 0.05 & 14.3218 \\
$\mathbf{1 0}$ & $5 \%$ & 5 & 5 & 0.05 & 17.5417 \\
$\mathbf{5}$ & $2 \%$ & 5 & 5 & 0.05 & 8.6691 \\
$\mathbf{5}$ & $5 \%$ & 5 & 5 & 0.05 & 14.3218 \\
$\mathbf{5}$ & $10 \%$ & 5 & 5 & 0.05 & 21.5525 \\
$\mathbf{5}$ & $5 \%$ & 2 & 5 & 0.05 & 14.017 \\
$\mathbf{5}$ & $5 \%$ & 5 & 5 & 0.05 & 14.3218 \\
$\mathbf{5}$ & $5 \%$ & 10 & 5 & 0.05 & 14.6208 \\
$\mathbf{5}$ & $5 \%$ & 5 & 2 & 0.05 & 13.7837 \\
$\mathbf{5}$ & $5 \%$ & 5 & 5 & 0.05 & 14.3218 \\
$\mathbf{5}$ & $5 \%$ & 5 & 10 & 0.05 & 14.194 \\
$\mathbf{5}$ & $5 \%$ & 5 & 5 & 0.02 & 14.3218 \\
$\mathbf{5}$ & $5 \%$ & 5 & 5 & 0.05 & 14.3106 \\
$\mathbf{5}$ & $5 \%$ & 5 & 5 & 0.1 & \\
\hline
\end{tabular}

Table 2- Nusselt Number $(\mathrm{Nu})$ for forced convection.

Tables $1 \& 2$ display the Nusselt number values with base of the plate. Thus rate of heat transfer in forced convection was more than counterpart with free convection. Inertial force reducing the heat transfer rate in natural convection but the enhancement of $\mathrm{Nu}$ observed in forced convection. The composition of metal particles enhances the heat transfer rate in both convections, which emphasizes the Nano-fluid significance. Lorentz forces enhance heat transfer rate slightly. Heat source obviously increase heat transfer rate in both convections. Thus rate of heat transfer was more when plate is stretched in free convection but no significance observed in forced convections.

\section{Conclusions}

1. Rate of heat transfer is more when plate stretched in free convection but no significance observed in forced convections. The increase of nano particle presence, increase of heat source enhances the heat transfer rate in forced convection than in free convection. This study reports that stretching parameter and nano particle concentration controls the heat transfer rate significantly.

2. It is evident that the vertical flow is inversely proportional to the stretching rate in natural convection and the flow is directly proportional to the stretching rate in forced convection.

3. The temperature is well controlled by stretch parameter and the nano particle in both convections. On the other hand heat source has significant effect in both convections. 
B.Sailaja et al.

International Journal of Thermofluid Science and Technology (2020), Volume 7, Issue 3, Paper No. 070302

\section{References}

[1] L.J. Crane, Flow past a stretching plate, ZAMM-Journal of Applied Mathematics and Physics, volume 21 (1970), pp: 645-647.

[2] P.S. Gupta, A.S. Gupta, Heat and mass transfer on a stretching sheet with suction or blowing, Canadian Journal of Chemical Engineering, volume 55 (1977), pp: 744-746.

[3] C.K. Chen, M.I. Char, Heat transfer of a continuous stretching surface with suction or blowing, Journal of Mathematical Analysis and Applications, volume 135(1988), pp: 568 580.

[4] A. Ishak, R. Nazar, I. Pop, Boundary layer flow and heat transfer over an unsteady stretching vertical surface, Meccanica, volume 44 (2009), pp: 369-375.

[5] B. McLeod, K.R. Raja gopal, On the non-uniqueness of the flow of a Navier Stokes fluid due to stretching boundary, Archive for Rational Mechanics and Analysis, volume 98 (1987), pp: 385-493.

[6] K.R. Rajagopal, T.Y. Na, A.S. Gupta, Flow of a visco-elastic fluid over a stretching sheet, Rheol Acta, volume 23 (1984), pp: 213-215.

[7] B. Siddappa, M.S. Abel, Non-Newtonian flow past a stretching plate, ZAMM-Journal of Applied Mathematics and Physics, volume 36 (1985), pp: 47-54.

[8] V.M. Soundalgekar, T.V. Ramanamurthy, Heat transfer past a continuous moving plate with variable temperature, Warme- und Stoffubertragung, volume 14 (1980), pp: 91-93.

[9] L.G. Grubka, K.M. Bobba, Heat transfer characteristics of a continuous stretching surface with variable temperature, ASME Journal of Heat Transfer, volume 107 (1985), pp: 248-250.

[10] M.E. Ali, Heat transfer characteristics of a continuous stretching surface, Warme und Stoffubertragung, volume 29 (1994), pp: 227-234.

[11] W.H.H. Banks, Similarity solutions of the boundary-layer equations for a stretching wall, Journal of Mechanical Theory and Applications, volume 2 (1983), pp: 375-392.

[12] B. Shankar Goud, B. Suresh Babu, MN Raja Shekar, G.Srinivas, Mass Transfer Effects on MHD Flow through Porous Medium past an Exponentially Accelerated Inclined Plate with Variable Temperature and Thermal Radiation, International journal of thermofluid science and technology, volume 6 (2020), pp: 1-14.

[13] K. Vajravelu, Convection heat transfer at a stretching sheet with suction or blowing, J. Math. Anal. Appl. vol. 188 (1994), pp: 1002-1011.

[14] T.S. Chen, F.A. Strobel, Buoyancy effects in boundary layer adjacent to a continuous moving horizontal flat plate, ASME Journal of Heat Transfer, volume 102 (1980), pp: 170-172.

[15] A. Moutsoglou, T.S. Chen, Buoyancy effects in boundary layers on inclined continuous moving sheets, ASME Journal of Heat Transfer, volume 102 (1980), pp: 371-373.

[16] O.D. Makinde, A. Aziz, MHD mixed convection from a vertical plate embedded in a porous medium with a convective boundary condition, International Journal of Thermal Sciences, volume 49 (2010), pp: 1813-1820.

[17] S. Kakaç, A. Pramuanjaroenkij, Review of convective heat transfer- enhancement with nanofluids, International Journal of Heat Mass Transfer, volume 52 (2009), pp: 3187-3196.

[18] S. Choi, Enhancing thermal conductivity of fluids with nanoparticle. In: D.A. Siginer, H.P. Wang (Eds.), Developments and Applications of Non- Newtonian Flows, MD volume 231 and FED volume 66, ASME 1995, pp: 99-105.

[19] H. Masuda, A. Ebata, K. Teramae, N. Hishinuma, Alteration of thermal conductivity and viscosity of liquid by dispersing ultra-fine particles, PLOS ONE, Netsu Bussei (Japan), volume 7 (1993), pp: 227-233.

[20] J. Buongiorno, W. Hu, Nanofluid coolants for advanced nuclear power plants, in: Proceedings of ICAPP'05, Seoul, Paper no. 5705 (May 15-19, 2005).

[21] N. Bachok, A. Ishak, R. Nazar, I. Pop, Flow and heat transfer at a general three dimensional stagnation point in a nanofluid, Physica B, volume 405 (2010), pp: 4914-4918. 
B.Sailaja et al.

International Journal of Thermofluid Science and Technology (2020), Volume 7, Issue 3, Paper No. 070302

[22] Sarit Kumar Das, Stephen U.S. Choi, Hrishikesh, E. Patel, Heat Transfer in Nanofluids - A Review, Heat Transfer Engineering, Vol 27(10), 2006, pp: 3-19.

[23] Xiang - Qi Wang, Arun S, Mujumdar, Heat Transfer Characteristics of Nanofluids: A Review, International Journal of Thermal Sciences, Vol 46, 2007, pp: 1-19.

[24] Tiwari Arun Kumar, Ghosh Pradyumna and Sarkar Jahar, Investigation of Thermal Conductivity and Viscosity of Nanofluids, Journal of Environmental Research and Development, Vol.7(2), 2012.

[25] Gbadeyan J.A, Olanrewaju M.A and Olanrewaju P.O, Boundary Layer Flow of a Nanofluid past a Stretching Sheet with a Convective Boundary Conduction in the Presence of Magnetic Field and Thermal Radiation, Australian Journal of Basic and Applied Sciences, Vol 5(9), 2011, pp: 1323-1334.

[26] M.A.A. Hamad and I. Pop, Unsteady MHD free convection flow past a vertical permeable flat plate in a rotating frame of reference with constant heat source in a Nanofluid, Heat Mass Transfer, Vol 47, 2011, pp:1517-1524.

[27] Hassan A.M, El-Areaway, Exact Solutions of Mass Transfer over a Stretching Surface with Chemical Reaction and Suction/ Injection, Journal of Mathematics and Statistics, Vol 5 (3), 2009, pp: 159- 166, ISSN: 1549-3644.

[28] G.V.P.N.Srikanth, B.Suresh Babu, G. Srinivas, Heat and Mass Transfer of a MHD Nanofluid with Chemical Reaction Effects, International Journal of Mechanical And Production Engineering, Volume 2(3), March-2014, ISSN: 2320-2092. 